\title{
Intradermal DNA Electroporation Induces Cellular and Humoral Immune Response and Confers Protection against HER2/neu Tumor
}

\author{
Alessia Lamolinara, ${ }^{1}$ Lorenzo Stramucci, ${ }^{1,2}$ Albana Hysi, ${ }^{1}$ Manuela Iezzi, ${ }^{1}$ \\ Cristina Marchini, ${ }^{3}$ Marianna Mariotti, ${ }^{1}$ Augusto Amici, ${ }^{3}$ and Claudia Curcio ${ }^{1}$ \\ ${ }^{1}$ Aging Research Center, G. d'Annunzio University, 66100 Chieti, Italy \\ ${ }^{2}$ Department of Medicine, Marlene and Stewart Greenebaum Cancer Center, University of Maryland School of Medicine, \\ Baltimore, MD 21201, USA \\ ${ }^{3}$ Department of Bioscience and Biotechnology, University of Camerino, Camerino, 63100 Macerata, Italy
}

Correspondence should be addressed to Claudia Curcio; c.curcio@unich.it

Received 19 March 2015; Revised 18 June 2015; Accepted 24 June 2015

Academic Editor: Peirong Jiao

Copyright ( $) 2015$ Alessia Lamolinara et al. This is an open access article distributed under the Creative Commons Attribution License, which permits unrestricted use, distribution, and reproduction in any medium, provided the original work is properly cited.

\begin{abstract}
Skin represents an attractive target for DNA vaccine delivery because of its natural richness in APCs, whose targeting may potentiate the effect of vaccination. Nevertheless, intramuscular electroporation is the most common delivery method for ECTM vaccination. In this study we assessed whether intradermal administration could deliver the vaccine into different cell types and we analyzed the evolution of tissue infiltrate elicited by the vaccination protocol. Intradermal electroporation (EP) vaccination resulted in transfection of different skin layers, as well as mononuclear cells. Additionally, we observed a marked recruitment of reactive infiltrates mainly 6-24 hours after treatment and inflammatory cells included CD $11 c^{+}$. Moreover, we tested the efficacy of intradermal vaccination against Her2/neu antigen in cellular and humoral response induction and consequent protection from a Her2/neu tumor challenge in Her2/neu nontolerant and tolerant mice. A significant delay in transplantable tumor onset was observed in both BALB/c $(p \leq 0,0003)$ and BALB-neuT mice $(p=0,003)$. Moreover, BALB-neuT mice displayed slow tumor growth as compared to control group $(p<0,0016)$. In addition, while in vivo cytotoxic response was observed only in BALB/c mice, a significant antibody response was achieved in both mouse models. Our results identify intradermal EP vaccination as a promising method for delivering Her2/neu DNA vaccine.
\end{abstract}

\section{Introduction}

DNA vaccination is an attractive immunotherapeutic strategy that triggers physiologic immunity and is able to induce long lasting T cell and antibody-mediated tumor protection [1]. In fact, direct injection into mouse muscle or skin of plasmid DNA encoding a selected antigen results in the expression of the gene product and can elicit an immune response against the antigen of interest [2]. Currently, various delivery devices such as gene-guns, bioinjectors, and electroporation systems are being used in order to increase the potency of DNA vaccines [3]. In vivo DNA electroporation (EP) has emerged as an efficient delivery method that allows efficient
DNA uptake, long-term and high-level antigen expression [4]. Furthermore, EP is also able to evoke the production of several cytokines and chemokines, thereby increasing the potency of DNA vaccines [4].

Muscle is the most commonly targeted tissue for evaluation of EP in combination with DNA delivery [5]. Under the influence of an electrical field, cellular membranes build up a transmembrane potential until the dielectric strength of the membrane is exceeded and permeation event occurs [6]. Intramuscular electroporation has been previously shown to induce target antigen expression and to trigger humoral and cellular immunity, thus enhancing tumor protection $[7,8]$. EP has been used clinically to deliver chemotherapeutic agents 
to tumor cells in cutaneous malignancies $[9,10]$. Nowadays, there are approximately 85 clinical trials listed using electroporation (http://www.clinicaltrials.gov/): around 28 are related to drug delivery and the rest are related to DNA delivery [11-13]. Moreover, several data establish EP as a potent method for stimulating immune responses induced by DNA vaccination in humans $[14,15]$.

Skin is an attractive site for electroporation in translational settings, as it is readily accessible and EP is minimally invasive and generally well tolerated [16], as compared to muscle. Moreover, skin naturally harbors a high number of antigen presenting cells (APCs), such as Langerhans cells and other types of dermal dendritic cells, which after DNA/antigen uptake can migrate to lymph nodes where efficient presentation to $\mathrm{T}$ cells occurs $[17,18]$, thereby potentially increasing EP efficacy.

The efficacy of intramuscular injection of a plasmid coding for the extracellular and transmembrane domains of the protein product of the Her-2/neu oncogene (ECTM) followed by EP in transgenic murine models has been previously demonstrated [10]. The vaccination protocol induced production of antibodies against Her- $2 /$ neu and IFN- $\gamma$ secretion: these two immune activities were associated with the clearance of Her-2/neu spontaneous lesions in transgenic BALB-neuT mice [10]. However, sequential courses of DNA intramuscular EP were required to maintain specific antibody response and counteract the progression of preneoplastic lesions to invasive carcinoma.

In the current study, we evaluated the effectiveness of intradermal vaccination using EP against transplantable Her $2 / \mathrm{neu}^{+}$tumor. To address this point, first we analyzed intradermal EP vaccination-induced immune cell recruitment, in both the skin and draining lymph nodes. Second, we evaluated, in both Her2/neu tolerant (BALB-neuT) and nontolerant $(\mathrm{BALB} / \mathrm{c})$ mice, the ability of intradermal ECTM EP vaccination to trigger Her2/neu specific immune responses and counteract tumor onset and growth.

\section{Material and Methods}

2.1. Mice. Seven-week-old virgin female BALB/c and BALBneuT mice $\left(\mathrm{H}-2^{\mathrm{d}}\right)$ were used. BALB/c mice were from Charles River Laboratories (Calco, Italy). Virgin BALB-neuT mice, overexpressing the transforming rat Her-2/neu oncogene under the control of the mouse mammary tumor virus [19], were bred in house. Mice of the same age were randomly assigned to experimental groups and were treated according to the European Community guidelines.

\subsection{Injection of Plasmids or FITC-Dextran and Electropora-} tion. pVAX (Invitrogen, Milan, Italy) was the backbone for all the vaccines. The cDNA sequence for ECTM was obtained as previously described [19]. The pVAX-EGFP DNA construct was obtained by subcloning the EGFP cDNA, excised from pEGFP-N1 (Clontech, Mountain View, CA) by HindIII/XbaI digestion, into the HindIII/XbaI sites of the $\mathrm{pVAX}-1$ vector (Life Technologies, Carlsbad, CA). The inserted sequence was verified by sequencing.
All plasmids for DNA immunizations were grown in E. coli DH5 $\alpha$ strain, and large-scale preparation of the endotoxin-free plasmid DNA was carried out using Qiagen EndoFree Plasmid-Giga kits (Qiagen, Chatsworth, CA, USA) according to the manufacturer's instructions. Endotoxin-free plastic ware was used to prevent recontamination of the plasmid.

Anesthetized mice were vaccinated with $50 \mu \mathrm{L}$ of solution containing $50 \mu \mathrm{g}$ of DNA or $25 \mathrm{mg} / \mathrm{mL}$ of fluorescein isothiocyanate-dextran (FITC-dextran) average mol wt 40,000 (Sigma Aldrich, Milan, Italy). The vaccination course consisted of two intradermal injections into the skin of the back near the base of the tail. Immediately after the plasmid or FITC-dextran administration, a conducting gel and an electrode were placed over the injection site and voltage was set up according to previously described protocols (2 pulses, $1125 \mathrm{~V} / \mathrm{cm} 50 \mu \mathrm{s}$, and 8 pulses, $275 \mathrm{~V} / \mathrm{cm} 10 \mathrm{msec}$ ) [20]. Electrodes conslehisted of two parallel lamellae at $6-8 \mathrm{~mm}$ distance. Pulses were generated by an Igea Cliniporator (Igea, Carpi, Italy). BALB/c and BALB-neuT mice were vaccinated 21 and 7 days before a subcutaneous injection with a lethal dose of TUBO cells (day 0 ).

2.3. Cell Lines. Her-2/neu positive TUBO cells had been originally isolated from a carcinoma arising in a BALB-neuT mouse [21]. N202.1A (Her-2/neu positive) and N202.1E (Her$2 /$ neu negative) lines had been isolated from a mammary carcinoma in a FVB/N mouse $\left(\mathrm{H}-2^{\mathrm{q}}\right)$ transgenic for the rat Her-2/neu protoncogene [22]. TUBO cells were cultured in DMEM (BioWhittaker Europe, Verviers, Belgium) with 20\% FBS (Life Technologies, Inc., San Giuliano, Italy), and N202.1A and N2021E in RPMI (BioWhittaker Europe) with 10\% FBS (Life Technologies).

2.4. Tumor Challenge. Mice were subcutaneously injected with $0.2 \mathrm{~mL}$ of a single cell suspension containing the minimal lethal dose of TUBO $\left(10^{5}\right)$ cells [19] in the right flank. Mice were checked twice weekly for tumor onset: stable or growing masses were regarded as tumors. Tumor growth was evaluated twice weekly in a blind fashion. Neoplastic masses were measured with calipers in the two perpendicular diameters, and the tumor volume was recorded for 150 days. At the end of this period, tumor-free mice were classified as survivors. Mice were euthanized when the tumor exceeded $1 \mathrm{~cm}^{3}$ volume for humane reasons.

2.5. Antibody Response. Sera were collected 14, 30, 80, and 100 days after vaccination. $100 \mu \mathrm{L}$ of $1: 10$ diluted sera was incubated for 45 minutes at $4^{\circ} \mathrm{C}$ with $2 \times 10^{5} \mathrm{~N} 2021 \mathrm{~A}$ or $1 \mathrm{E}$ cells pretreated with Fc receptor blocker (CD16/CD32; Pharmingen, Milan, Italy) for 15 minutes at $4^{\circ} \mathrm{C}$. Total $\mathrm{Ig}$ binding was evaluated using a PE-conjugated goat anti-mouse Ig antibody (DakoCytomation, Milan, Italy). The Ab4 (Oncogene, Milan, Italy) was used as positive control for anti-Her2/neu reactivity. Results were expressed as mean fluorescence intensity (MFI) [23]. N202.1E MFI was subtracted to N202.1A MFI to identify Her2/neu specific antibody reactivity and differences in MFI were analyzed by Student's $t$-test. 
Isotype determination was carried out by an indirect immunofluorescence procedure. Dilutions $(1: 10)$ of sera in PBS-azide-BSA were incubated for 45 minutes at $4^{\circ} \mathrm{C}$ with 2 $\times 10^{5} \mathrm{~N} 202.1 \mathrm{~A}$ or N202.1E cells, pretreated with Fc receptor blocker (CD16/CD32; Pharmingen) for 15 minutes at $4^{\circ} \mathrm{C}$. After washing, the cells were incubated for 30 minutes with rat Alexa 488-conjugated antibodies anti-mouse IgM, IgG1, IgG2a, IgG2b, or IgG3 (Invitrogen) and fluorescence evaluated. The specific N202.1A-binding potential was calculated as the percentage of MFI/total Ig.

Flow cytometry experiments were performed using a FACSCalibur (Becton Dickinson, Milan, Italy) and analyzed with FlowJo software (FlowJo LLC, Ashland, USA).

2.6. In Vivo Cytotoxicity Assay. In vivo cytotoxicity assay was performed as previously described [24], with slight modifications. Briefly, a single-cell suspension of $10^{7}$ naive splenocytes $(\mathrm{Spc}) / \mathrm{mL}$ was labeled with $0.5\left(\mathrm{CFSE}^{\text {low }}\right)$ or $5 \mu \mathrm{mol} / \mathrm{L}$ (CFSE $^{\text {high }}$ ) of the carboxyfluorescein diacetate succinimidyl ester CFSE (Molecular Probes, Leiden, Netherlands). Spc labeled with $5 \mu \mathrm{mol} / \mathrm{L}$ CFSE were also pulsed with $15 \mu \mathrm{g} / \mathrm{mL}$ of $\mathrm{H}-2 \mathrm{~K}$ dominant Her2/neu (TYVPANASL) (Inbios SRL, Naples, Italy) peptide for 1 hour at room temperature. The two Spc populations were mixed together in equal amounts and injected i.v. into control and treated mice. Mice were sacrificed 48 hours later, and single-cell suspensions from spleens were processed individually to evaluate the presence of CFSE ${ }^{\text {high }}$ cells with the FACSCalibur after adding propidium iodide to exclude dead cells. The specific cytolytic activity was calculated as $100 \times$ (percentage CFSE ${ }^{\text {low }}$ cells percentage CFSE $^{\text {high }}$ cells)/percentage CFSE $^{\text {low }}$ cells.

2.7. Immunohistochemistry and Immunofluorescence. For immunohistochemical evaluation, mice were euthanized at $3,6,24,48,72$, and 96 hours after vaccination and the skin and axillary, inguinal, and popliteal lymph nodes were collected, fixed in PFA 1\%, and embedded in OCT and tissue sections were stained with hematoxylin and eosin (H\&E) for histological examination. Skin and lymph nodes sections were incubated with the following primary antibodies: rat $\mathrm{mAb}$ anti-CD4, anti-CD8 $\alpha$, anti-CD11b, anti-CD45R/B220, anti-Gr-1 (BD Pharmingen, Milan, Italy), anti-CD68 (Abcam, Cambridge, UK), and anti-CD1lc (Chemicon International, USA). After washing, slides were overlaid with appropriate secondary antibodies. Immunostaining was developed with Vulcan Fast Red (Biocare, Milan, Italy) alkaline phosphatase method. For immunofluorescence, secondary antibodies conjugated with Alexa 488 and Alexa 546 (Invitrogen, Life Technologies, Monza, Italy) were used. Nuclei were stained with DRAQ5 (Alexis, Life Technologies, Monza, Italy), YO PRO-3, or TO PRO-3 (Molecular Probes, Monza, Italy). Image acquisition was performed using Zeiss LSM 510 Meta confocal microscope (Carl Zeiss SpA, Milan Italy).

The slides were examined in a double-blind fashion, and digital images of representative areas were taken.
2.8. Statistics. Quantitative data are presented as mean \pm SEM from three independent experiments. The significance of differences was evaluated with two-tailed Student's $t$-test or log-rank (Mantel-Cox) test. Statistical analysis was carried out with GraphPad Prism5 Software (San Diego, CA, USA); $p \leq 0.05$ was used as the critical level of significance.

\section{Results}

3.1. Intradermal EP Vaccination Results in a Marked Recruitment of Reactive Infiltrates. To analyze the cellular effects of vaccination, intradermal injection of FITC-dextran followed by $\mathrm{EP}$ was performed in BALB/c mice. Immediately after vaccination (3 hours) skin showed tissue damage and a coagulated aspect as well as evident inflammatory infiltrate (Figure 1(a)). Immunofluorescence analysis revealed the presence of CD11b macrophages with incorporated FITCdextran (Figures 1(b)-1(d)) in close proximity of the damaged area as well as in epidermis (Figure 1(b)), dermis (Figure 1(c)), and hypodermis (Figure 1(d)). At this extremely early time point, no cells with encapsulated FITC-dextran were detected in lymph nodes (not shown). At a later time point (6 hours after vaccination), CD1lc cells with incorporated FITC-dextran were detected in the dermis (Figure 1(e)). Additionally, FITC-dextran was also incorporated by Lyve 1 lymphatic endothelial cells (Figure 1(f)) and deposited outside CD31 blood vessels (Figure 1(g)). Some cells with incorporated FITC-dextran were found into inguinal lymph nodes (draining lymph nodes) (Figure $1(\mathrm{~h})$ ). No FITCdextran incorporating cells were detected in control mice intradermally injected without electroporation. Altogether these data are consistent with an efficient transfection of APCs and their progressive migration through the skin layers to lymph nodes.

Accordingly, immunohistochemistry analysis highlighted the accumulation of granulocytes (Grl) and macrophages (CD11b) 3 hours after treatment (Table 1) and a significant number of reactive cells were still observed at 6 hours. In particular, at this time point, dendritic cells (CD11c) and $\mathrm{T}$ lymphocytes (CD4) were detected (Table 1 and Figures 2(a) and 2(b)). Twenty-four hours after vaccination the number of dendritic and CD4 cells decreased until almost baseline levels (Table 1 and Figures 2(c) and 2(d)) and the local inflammatory infiltrate gradually turned off starting at 48 hours after vaccination.

In order to shed light into the mechanisms underlying the immune responses elicited by the intradermal vaccination, we analyzed the type and the localization of pVax EGFP transfected cells in both skin and inguinal draining lymph nodes (Figure 3). After an intradermal injection probably the major part of the volume is deposited in the dermis due to the epidermis being very thin, particularly in mice. However, twenty-four hours after vaccination, positive cells have also been found in epidermis and in the subcutaneous muscle layer, the panniculus carnosus, near the damaged zone (Figure 3(a)). Close to them, some infiltrating cells expressed the transgene too (Figure 3(a)). As shown in Table 1, dendritic cells decreased in skin 24 hours after treatment, so we investigated the presence of these cells in draining lymph nodes. 


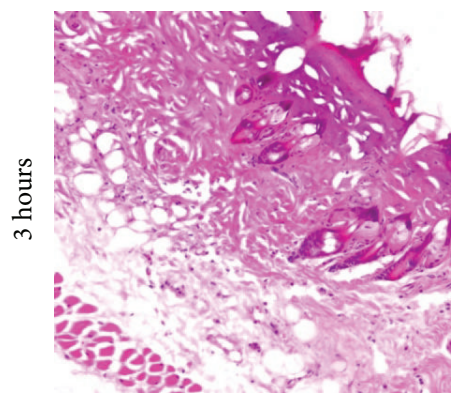

(a)

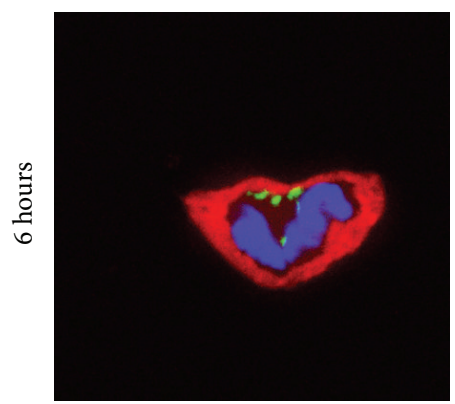

(e)

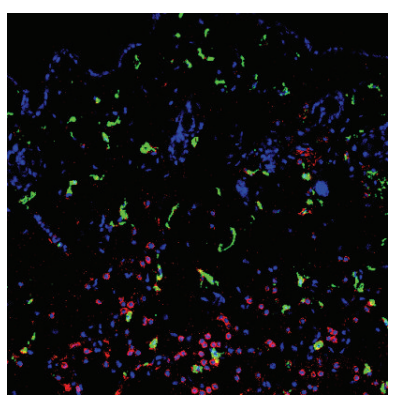

(b)

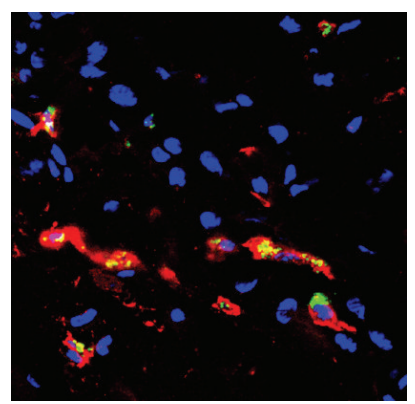

(f)

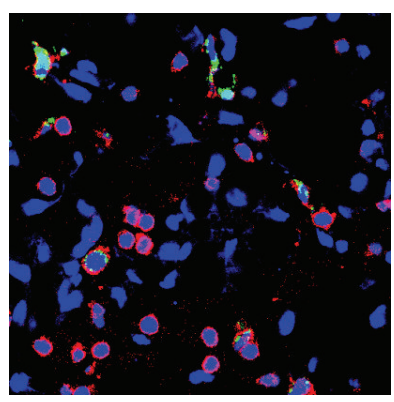

(c)

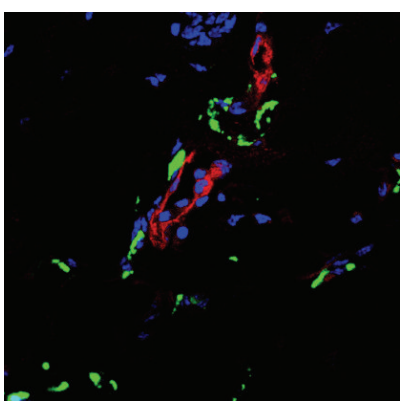

(g)

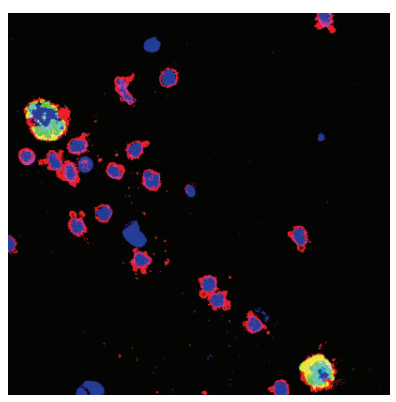

(d)

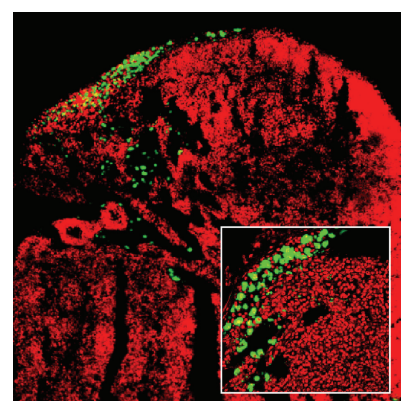

(h)

FIGURE 1: Electroporation effect on FITC-dextran expression and distribution in skin. Three hours after intradermal injection of FITC-dextran followed by EP reactive cells that are recruited (a). Among these CD11b (red) cells incorporate FITC-dextran and are found in different skin layers ((b), (c), (d)). Six hours after vaccination, CD11c (red (e)) Lyve 1 (lymphatic vessel, red (f)) and CD31 (endothelial vessels, red (g)) cells are detected in the skin. FITC-dextran and nuclei are shown in green and blue, respectively $((\mathrm{b})-(\mathrm{g}))$. FITC-dextran incorporating cells are observed in draining lymph nodes 6 hours after treatment (h). Nuclei are shown in red (h). Images are representative of three independent experiments. Magnification: (a), (b) $\times 200$; (c), (d), (f), (g) $\times 630$; (e) $\times 1500$; (h) $\times 200$, insert $\times 400$.

TABLE 1: Semiquantitative analysis of inflammatory cells present in the skin of BALB/c mice from 3 to 96 hours after pVAX-EGFP vaccination followed by electroporation.

\begin{tabular}{lccccccc}
\hline $\begin{array}{l}\text { Time after } \\
\text { intradermal } \\
\text { EP vaccination }\end{array}$ & Gr-1 & CD11b & CD68 & CD11c & CD4 & B220 & CD8 \\
\hline $3 \mathrm{~h}$ & +++ & +++ & ++ & + & + & $-/+$ & - \\
$6 \mathrm{~h}$ & ++ & +++ & ++ & ++ & +++ & + & $-/+$ \\
$24 \mathrm{~h}$ & ++ & ++ & + & + & ++ & + & $-/+$ \\
$48 \mathrm{~h}$ & + & ++ & + & $-/+$ & + & ++ & - \\
$72 \mathrm{~h}$ & + & ++ & + & $-/+$ & + & ++ & - \\
$96 \mathrm{~h}$ & $-/+$ & ++ & + & $-/+$ & $-/+$ & ++ & - \\
\hline
\end{tabular}

The expression of reactive cells was defined as absent $(-)$, scarcely $(-/+)$, moderately $(+)$, frequently $(++)$, or strongly $(+++)$ present on cryostat sections stained with the antibodies.

Not only transfected dendritic cells were found (Figure 3(b)), but also, in accordance with their APC role, these cells were transfected and in very tight proximity with both CD4 (Figure 3(c)) and B220 (Figure 3(d)).

\subsection{Intradermal EP Vaccination Protects against a Lethal} Tumor Challenge by Inducing Her2/neu Specific CTLs and Antibody Response. Intradermal ECTM EP vaccination effectively delayed tumor onset in both Her2/neu nontolerant and tolerant mice. In fact, while mice vaccinated with an empty plasmid developed tumor within 30 days (Figures 4(a) and $4(\mathrm{c})$ ), in pVAX-ECTM treated group, all BALB/c mice remained tumor free until the endpoint (Figures $4(\mathrm{a})$ and 4(b)), and BALB-neuT mice displayed a strong delay in tumor onset $(p=0,003)$ (Figure $4(c))$.

Notably, tumor growth in these mice was characterized by very slow kinetics as compared to pVAX group $(p<0,0016)$ (Figure 4(d)).

In accordance with the observed total protection against tumor challenge, higher CTL activity was observed in $\mathrm{BALB} / \mathrm{c}$ mice electroporated with pVAX-ECTM plasmid ( $p=$ $0,0369)$, while no cytotoxicity was detected in Her2/neu tolerant BALB-neuT mice (Figure 5(a)). This data together with a higher protection observed in Balb-c mice is consistent with a higher immune tolerance of BALB-neuT mice.

Moreover, 14, 30, and 80 days after last vaccination, the titer of anti-Her2/neu antibody was evaluated. A significant Her2/neu specific antibody titer was detected in both BALB/c $(p=0,0256)$ and BALB-neuT $(p=0,0096)$ pVAX-ECTM vaccinated mice as compared to pVAX groups (Figures 5(b) and 5(c)) already after 14 days. Interestingly the titer remained elevated also 30 days after the vaccination (BALB/c $p=0,0242$, BALB-neuT $p=0,0197$ ), with detectable high levels up to 100 days after vaccination (not shown) in all surviving mice. The induction and persistence of the humoral response in both mouse models, together with the similar isotype profiling of anti-Her2/neu reactive antibodies 


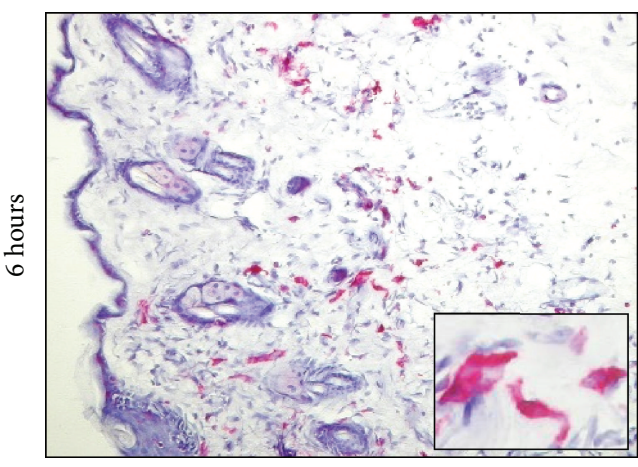

(a)

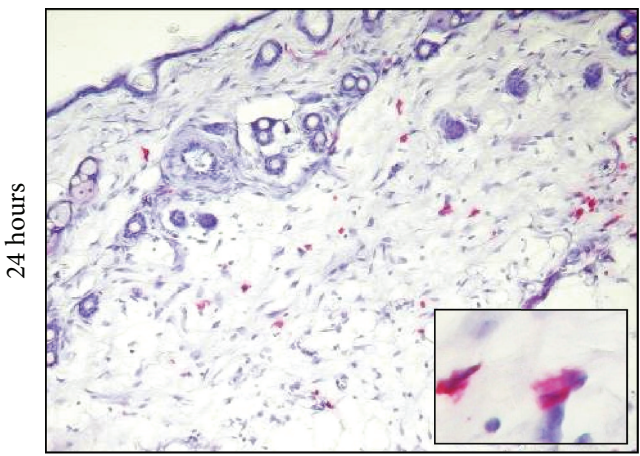

(c)

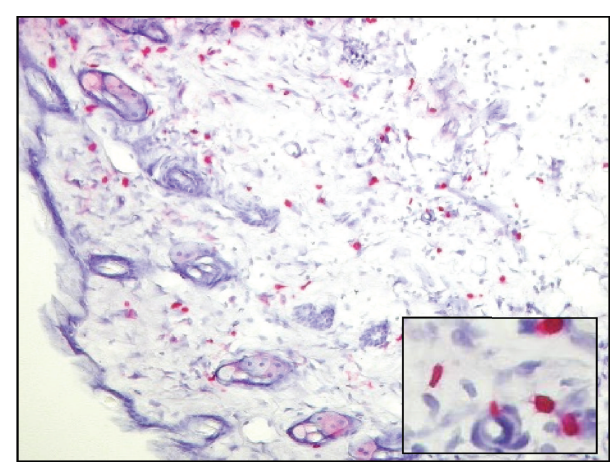

(b)

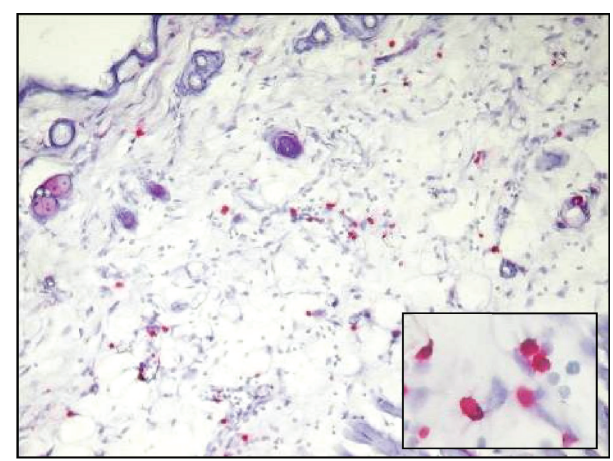

(d)

FIGURE 2: APCs infiltrate vaccinated skin. Six hours after intradermal EP vaccination dendritic (a) and CD4 (b) cells infiltrate the electroporated skin and decrease starting at 24 hours after treatment ((c), (d)). Red staining shows positivity for CD11 ((a), (c)) or CD4 ((b), (d)) molecule. Magnification: $\times 200$, insets: $\times 400$. Images are representative of three independent experiments.

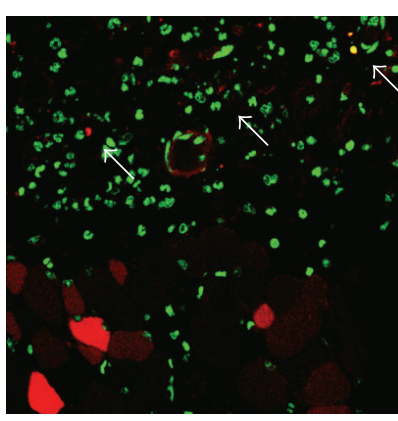

(a)

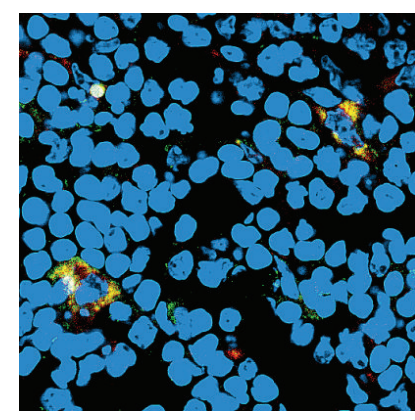

(b)

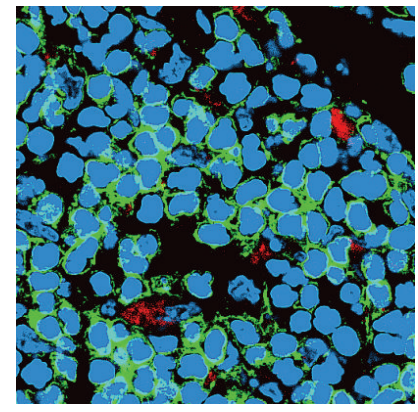

(c)

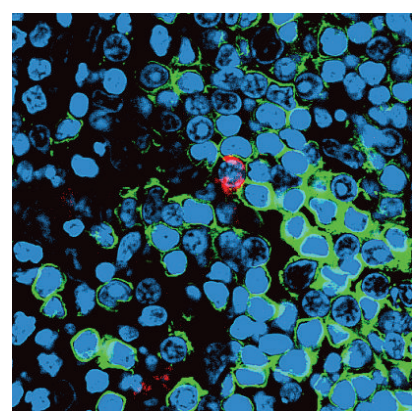

(d)

FIGURE 3: Intradermal EP vaccination transfects dendritic cells that migrate to draining lymph nodes. Transfected (red) muscle fibers and cells (arrows) in skin. Nuclei are shown in green. Magnification $\times 400$. (a) Transfected dendritic cells have migrated into lymph nodes (CD1lc in green, EGFP in red). (b) Interaction of dendritic cells that have incorporated EGFP (in red) with CD4 cells (in green) (c). Transfected cells (in red) adjacent to B220 cells (in green) in draining lymph node. (d) Nuclei are shown in blue, magnification $\times 630$. ((b)-(d)) Images are representative of three independent experiments.

(Figures 5(d) and 5(e)), indicate that this type of immunity is similarly triggered in both tolerant and nontolerant mice. Interestingly a high antibody titer correlated with tumor protection in BALB-neuT mice (see Supplementary Figure 1 in Supplementary Material available online at http://dx.doi.org/10.1155/2015/159145). It is important to note that naked DNA injection resulted in negligible antibody or cytotoxicity induction and tumor protection (Supplementary Figure 2).
Altogether these results indicate that our vaccination protocol is able to induce a protective immune response not only in Her-2 nontolerant BALB/c mice, but also in tolerant BALB-neuT mice.

\section{Discussion}

By virtue of its versatility and efficacy in inducing immunity against selected antigens, DNA vaccination represents a 


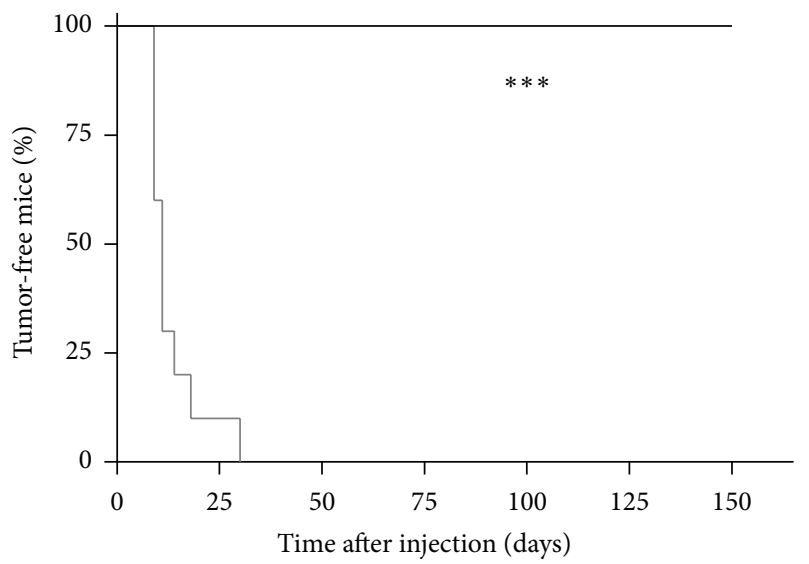

(a)

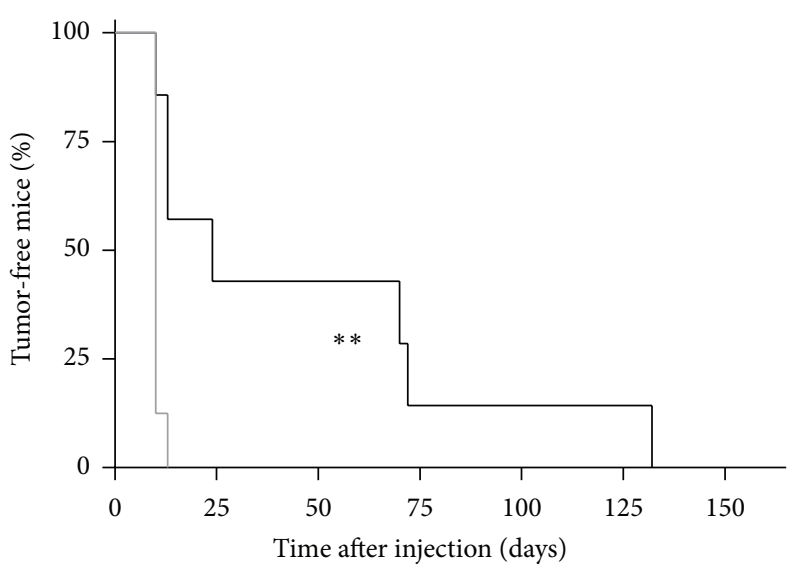

(c)

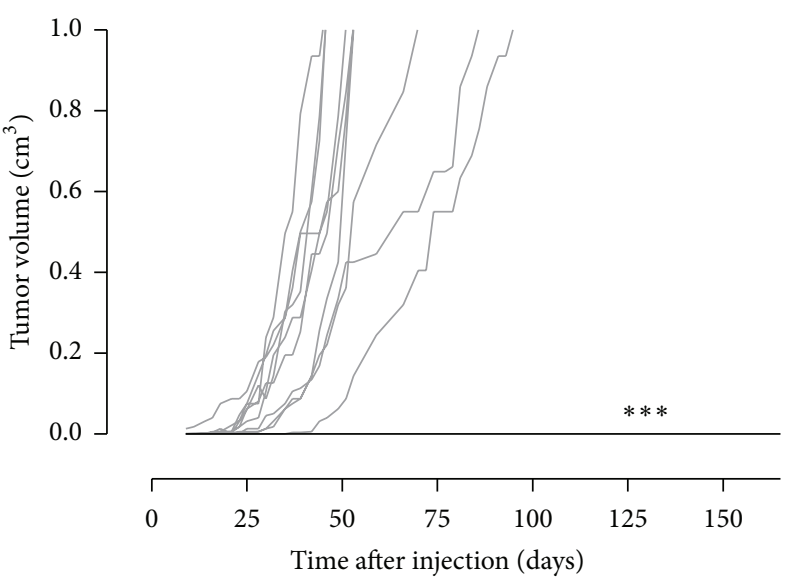

(b)

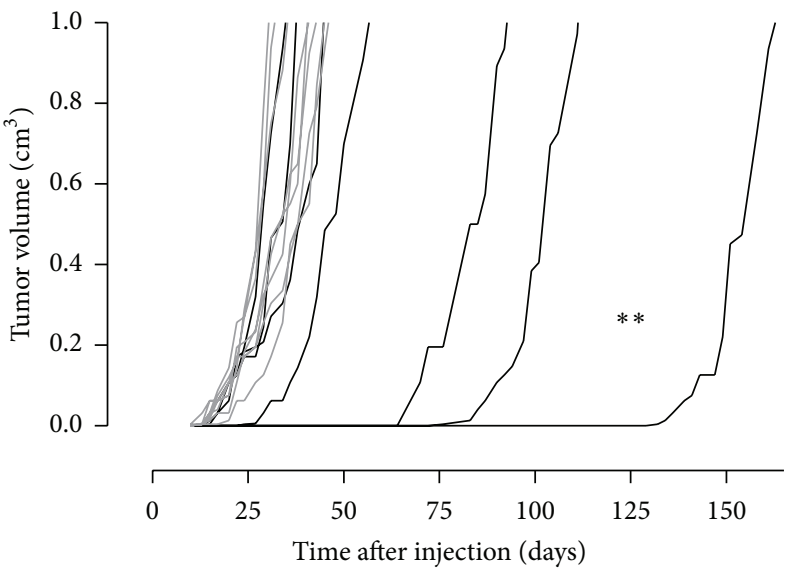

(d)

Figure 4: Intradermal EP vaccination protects BALB/c and BALB-neuT mice against a TUBO challenge. Mice were immunized twice with empty (pVAX, gray line) or ECTM (pVAX-ECTM, black line) plasmid. pVAX-ECTM treated BALB/c mice $(n=12)$ display total tumor protection $((\mathrm{a}), p<0,0001)$, while control group $(n=10)$ develops fast growing tumors within 30 days after challenge $((\mathrm{b}), p=0,0003)$. Vaccinated BALB-neuT mice $(n=7)$ display a delayed tumor onset $((\mathrm{c}), p=0,0030)$ and slower tumor growth $(p<0,0016)$ with respect to control group $(n=8)$. Each line refers to an individual tumor. Log-rank (Mantel-Cox) test and Student's $t$-test were used for statistical analysis.

suitable approach and may provide new therapeutic avenues for the treatment of tumors [25]. Plasmids can be delivered by intramuscular, intradermal/epidermal, subcutaneous, oral (e.g., with bacterial carrier), and pulmonary (e.g., aerosols) or other routes (e.g., vaginal) [26]. Notably, the skin is a particularly attractive site for vaccination given that intradermal vaccination is easy to perform and well tolerated both clinically and histopathologically [17]. Additionally, skin is characterized by an extended local network of dendritic cells and the easy access to the skin-draining lymph nodes generates effector $\mathrm{T}$ cells and immunoglobulin-producing $\mathrm{B}$ cells $[27,28]$. These characteristics may help the generation of a long-term protective immunity [29, 30]. In fact, experiments with bone marrow chimeras have shown that APCs, presumably dendritic cells, have a key role in DNA vaccination-induced protection [26]. Moreover, it is already known that using intradermal EP vaccination a wide variety of cell types can be transfected and these cells can be found in the draining afferent lymph nodes, suggesting that migration of directly transfected dendritic cells may occur [17]. Nevertheless, intradermal vaccination is notorious for its lower antibody responses compared to intramuscular route of plasmid administration in mice [31-34].

In this study we demonstrate that intradermal in vivo application of pulsed electric field confers long-term protection from Her2/neu tumor. Indeed, we report that efficiently permeabilized cells are found in all layers of the skin and migrate to draining lymph nodes. This is consistent with previous studies showing that, in contrast to the predominantly epidermal injection of naked DNA [35], a pattern of dermal and subdermal transfection is observed in the skin [10]. Concordantly with our data (Figures 1 and 2) transfected cell types included numerous mononuclear cells with dendritic morphology, as well as large numbers of adipocytes [17]. The variety of transfected cell types is likely to grant the mounting of an effective and durable immune response: in fact it has 


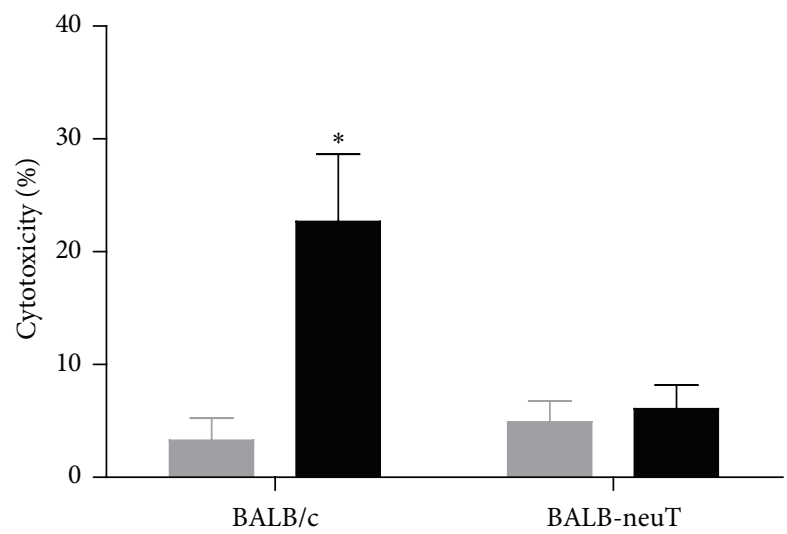

(a)

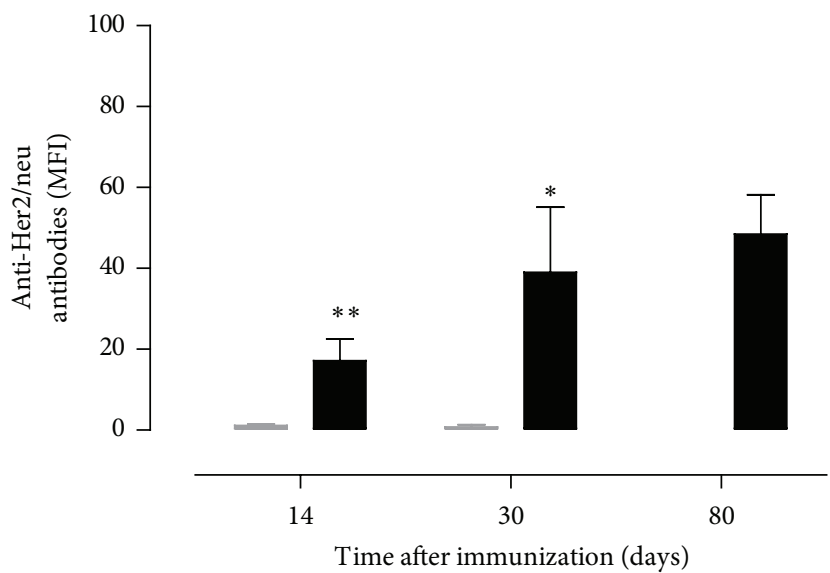

(c)

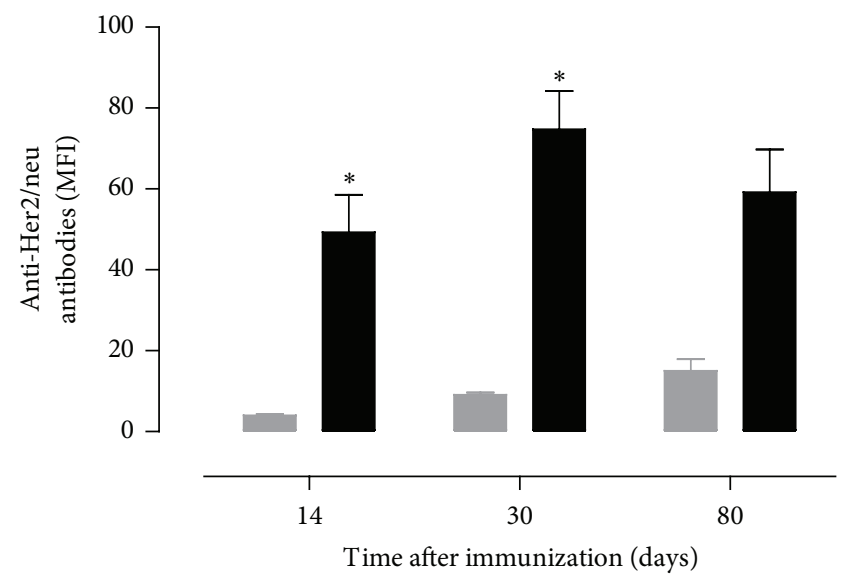

(b)

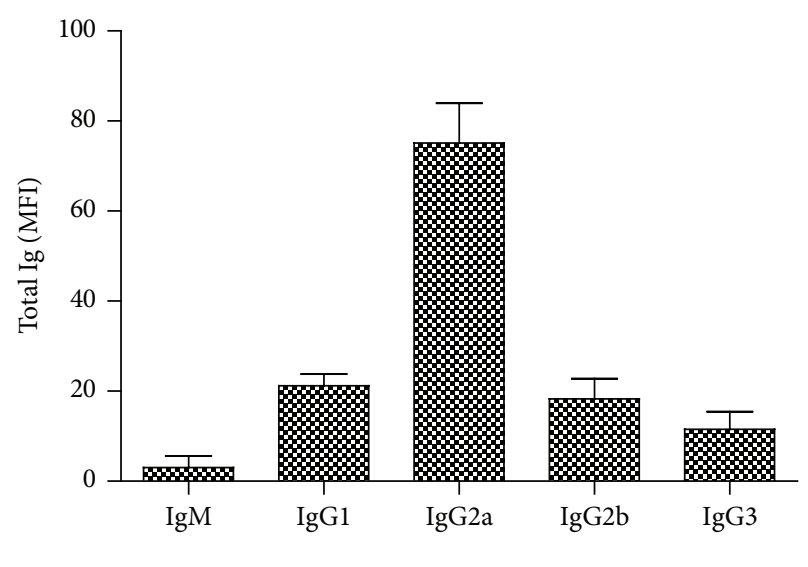

(d)

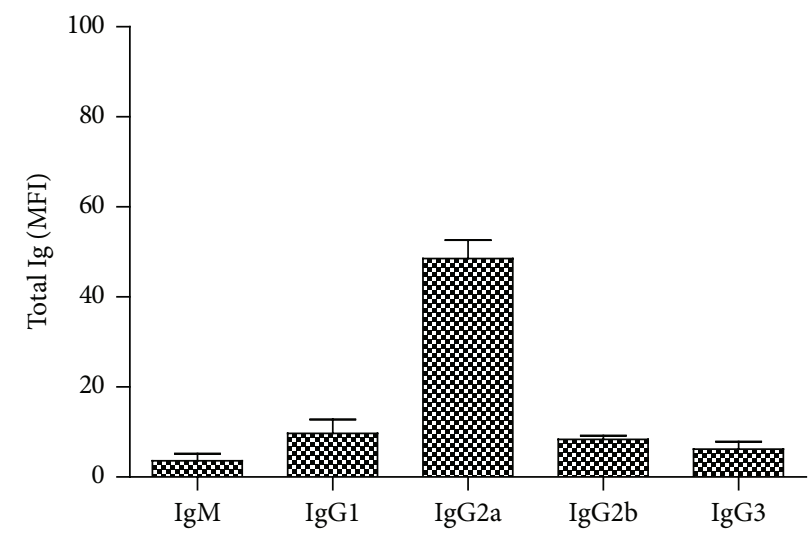

(e)

FIGURE 5: Intradermal EP vaccination induces cellular response in Her2/neu nontolerant mice and humoral immune response in both tolerant and nontolerant mice. In vivo cytotoxicity against the $\mathrm{H}-2^{\mathrm{d}}$ dominant peptide in BALB/c and BALB-neuT (each experimental group $n=3$ ) mice vaccinated with pVAX (gray bars) or pVAX-ECTM plasmid (black bars). Data are reported as $\%$ of lysis. ${ }^{*} p=0,0369$, Student's $t$-test. (a) Anti-Her2/neu antibody titer (MFI \pm SEM) in BALB/c (b) and BALB-neuT mice (c). ${ }^{* *} p=0,0096,{ }^{*} p<0,0242$, Student's $t$-test ((b), (c)). Isotype titration, performed in three different pools of sera, revealed similar composition of Her2/neu specific antibodies for BALB/c (d) and BALB-neuT (e) treated mice ((d), (e)). 
been demonstrated that transfection of different cell types results into different immune responses [36-39].

We further demonstrated that CD11c are efficiently permeabilized and hopefully transfected upon DNA vaccination (Figure 3 and Supplementary Figure 2) as a result of electroporation. The preferential EP mediated transfection of cells with dendritic morphology could provide a distinct advantage due to the central role of such cells in the stimulation of both primary and secondary immune responses [17]. Dendritic cells are the key initiators and regulators of any immune response which determine the outcome of CD4 and CD8 $\mathrm{T}$ cell responses [30]. Nevertheless, given the fact that either direct transfection of APCs or uptake of protein from other transfected cells (cross presentation) [26] may occur, we cannot provide formal proof that, although efficiently permeabilized, skin APCs are directly transfected. However, either pathway may lead to an effective protection. In fact it has been demonstrated that, in response to vaccination, the cutaneous immune system sets up complex mechanisms including (1) recognition and capture of the vaccine by skinresident APCs or by recruited innate immune cells (inflammatory microenvironment), (2) activation of skin APCs, (3) passive diffusion or cell transport of antigens to the secondary lymphoid organs draining the skin (draining lymph nodes), (4) processing and presentation of the antigens to immature CD4 and CD8 T cells by activated APCs or to naive follicular $\mathrm{B}$ cells, (5) activation of antigen-specific CD4 and CD8 T cells and clonal expansion as well as activation of specific $B$ cells within a germinative center, (6) migration of specific effector CD4 and CD8 T and B cells toward the zone of vaccination and elimination of the vaccine antigens, (7) generation of a pool of specific memory T and B cells in the secondary lymphoid organs and in the periphery (skin and mucosa) [30].

Indeed, in our study, after intradermal EP vaccination, a significant number of reactive cells were already observed at 6 hours after treatment, consisting primarily of dendritic cells.

Independent on the mechanism by which APCs acquire and then present the target antigen, to the best of our knowledge, this is the first report showing that intradermal DNA injection targeting Her2/neu followed by EP elicts a strong inflammatory-like response and induces both CTL and antibody responses in BALB/c and humoral response in tolerant BALB-neuT mice. This is consistent with recent reports of the presence of both CTL and antibody responses after vaccination in other tumor models [40, 41]. A previous study suggested that intradermal plasmid injection could not induce an effective immune response in Her2/neu models [25]. Nevertheless, our study demonstrates that, similar to what have been reported for intramuscular route, electroporation boosts the efficacy of ECTM vaccination when intradermally administered (Supplementary Figure 1), resulting in a specific immunity and tumor protection. Additionally, previous studies demonstrated that, in BALB-neuT mice, intramuscular ECTM injection followed by EP repeated every 10 weeks triggered a humoral response that inhibited the progression of spontaneous mammary carcinogenesis [10]. Although we cannot directly compare the two methods, in our study, a single cycle of intradermal EP vaccination induced a significant delay in tumor onset with an antibody titre that remains high over time, indicating intradermal route as a valid alternative to induce Her2/neu specific response. This data confirms previous studies implicating anti-Her2/neu antibodies in the inhibition of Her2/neudriven carcinogenesis and progression [7, 22, 42, 43]. The importance of antibodies is further underlined by the reported absence of protection in BALB-neuT/ $\mu \mathrm{KO}$ mice unable to produce antibodies to Her2/neu after intramuscular DNA vaccinations with ECTM plasmid [30]. Moreover, the high titer of anti-Her2/neu antibodies has been demonstrated to correlate with the downregulation of Her2/neu in the cells of mammary lesions and its cytoplasmic confinement $[21,43,44]$. Consistent with a central role of antibodymediated protection [45-48] we observed protection from tumor challenge in BALB-neuT mice even in the absence of cell cytotoxicity, and long-term survivor mice displayed the highest antibody titer (Supplementary Figure 2).

Notably in our study we were able to achieve a longterm protection not only in Her2/neu nontolerant BALB/c mice that are more prone to mount an effective immune response against the oncogene (as demonstrated also by the capability of our vaccination to induce cytotoxicity in these mice), but also in highly tolerant BALB-neuT mice. BALB-neuT mice are genetically predestined to develop lethal invasive carcinomas in their mammary glands by week 22 [10]. As the transforming (and immunization target) oncogene is embedded in the genome of these mice, a unique dynamic relationship between the oncogenic signals and inhibitory immune reactions is taking place. Given the longterm protection achieved with only 2 vaccination rounds, it will be of interest to test our experimental setting on spontaneous Her2/neu positive mammary tumors to evaluate if intradermal $\mathrm{EP}$ vaccination can reduce the number of doses and/or prolong the tumor-free mice. In fact, prolongation of immune memory could be even a more worthwhile goal than enhancement of the immune response [42]. Indeed, activating the appropriate arm of the immune system that induces protection is now one of the major challenges to be faced in vaccine development against tumors. However, further studies are necessary to find out the best settings for vaccination, such as the nature of the antigens, against which immune responses are elicited, the use of adjuvant, and specific delivery system that will ensure optimal presentation to immune system and protection from tumors.

\section{Conflict of Interests}

The authors declare that there is no conflict of interests regarding the publication of this paper.

\section{Authors' Contribution}

Alessia Lamolinara and Lorenzo Stramucci equally contributed to this work.

\section{References}

[1] S. Gurunathan, D. M. Klinman, and R. A. Seder, "DNA vaccines: immunology, application, and optimization," Annual Review of Immunology, vol. 18, pp. 927-974, 2000. 
[2] D.-C. Tang, M. DeVit, and S. A. Johnston, "Genetic immunization is a simple method for eliciting an immune response," Nature, vol. 356, no. 6365, pp. 152-154, 1992.

[3] H. Davtyan, A. Hovakimyan, K. Zagorski et al., "BTX AgilePulse system is an effective electroporation device for intramuscular and intradermal delivery of DNA vaccine," Current Gene Therapy, vol. 14, no. 3, pp. 190-199, 2014.

[4] A. Luxembourg, C. F. Evans, and D. Hannaman, "Electroporation-based DNA immunisation: translation to the clinic," Expert Opinion on Biological Therapy, vol. 7, no. 11, pp. 1647-1664, 2007.

[5] A.-K. Roos, S. Moreno, C. Leder, M. Pavlenko, A. King, and P. Pisa, "Enhancement of cellular immune response to a prostate cancer DNA vaccine by intradermal electroporation," Molecular Therapy, vol. 13, no. 2, pp. 320-327, 2006.

[6] J. J. Drabick, J. Glasspool-Malone, S. Somiari, A. King, and R. W. Malone, "Cutaneous transfection and immune responses to intradermal nucleic acid vaccination are significantly enhanced by in vivo electropermeabilization," Molecular Therapy, vol. 3, no. 2, pp. 249-255, 2001.

[7] E. Quaglino, M. Iezzi, C. Mastini et al., "Electroporated DNA vaccine clears away multifocal mammary carcinomas in her2/neu transgenic mice," Cancer Research, vol. 64, no. 8, pp. 2858-2864, 2004.

[8] C. Curcio, A. S. Khan, A. Amici et al., "DNA immunization using constant-current electroporation affords long-term protection from autochthonous mammary carcinomas in cancerprone transgenic mice," Cancer Gene Therapy, vol. 15, no. 2, pp. 108-114, 2008.

[9] R. Heller, M. J. Jaroszeski, D. S. Reintgen et al., "Treatment of cutaneous and subcutaneous tumors with electrochemotherapy using intralesional bleomycin," Cancer, vol. 83, no. 1, pp. 148-157, 1998.

[10] R. Heller, R. Gilbert, and M. J. Jaroszeski, "In vivo gene delivery," Advanced Drug Delivery Reviews, vol. 35, pp. 131-137, 1999.

[11] L. C. Heller, Non-Viral Vectors for Gene Therapy: Physical Methods and Medical Translation, Chapter in Book, Academic Press, 2015.

[12] S.-H. Lee, S. N. Danishmalik, and J.-I. Sin, "DNA Vaccines, electroporation and their applications in cancer treatment," Human Vaccines \& Immunotherapeutics, 2015.

[13] B. Yang, J. Jeang, A. Yang, T. C. Wu, and C.-F. Hung, "DNA vaccine for cancer immunotherapy," Human Vaccines \& Immunotherapeutics, vol. 10, no. 11, pp. 3153-3164, 2014.

[14] A. I. Daud, R. C. De Conti, S. Andrews et al., "Phase I trial of interleukin-12 plasmid electroporation in patients with metastatic melanoma," Journal of Clinical Oncology, vol. 26, no. 36, pp. 5896-5903, 2008.

[15] L. Low, A. Mander, K. McCann et al., "DNA vaccination with electroporation induces increased antibody responses in patients with prostate cancer," Human Gene Therapy, vol. 20, no. 11, pp. 1269-1278, 2009.

[16] K. E. Broderick, A. S. Khan, and N. Y. Sardesai, "DNA vaccination in skin enhanced by electroporation," Methods in Molecular Biology, vol. 1143, pp. 123-130, 2014.

[17] J.-F. Nicolas and B. Guy, "Intradermal, epidermal and transcutaneous vaccination: from immunology to clinical practice," Expert Review of Vaccines, vol. 7, no. 8, pp. 1201-1214, 2008.

[18] K. K. Peachman, M. Rao, and C. R. Alving, "Immunization with DNA through the skin," Methods, vol. 31, no. 3, pp. 232-242, 2003.
[19] K. Boggio, G. Nicoletti, E. Di Carlo et al., "Interleukin 12mediated prevention of spontaneous mammary adenocarcinomas in two lines of HER-2/neu transgenic mice," Journal of Experimental Medicine, vol. 188, no. 3, pp. 589-596, 1998.

[20] A. Donate and R. Heller, "Assessment of delivery parameters with the multi-electrode array for development of a DNA vaccine against Bacillus anthracis," Bioelectrochemistry, vol. 94, pp. 1-6, 2013.

[21] S. Rovero, A. Amici, E. Di Carlo et al., "DNA vaccination against rat Her-2/Neu p185 more effectively inhibits carcinogenesis than transplantable carcinomas in transgenic BALB/c mice," The Journal of Immunology, vol. 165, no. 9, pp. 5133-5142, 2000.

[22] P. Nanni, G. Nicoletti, C. De Giovanni et al., "Combined allogeneic tumor cell vaccination and systematic interleukin 12 prevents mammary carcinogenesis in HER-2/neu transgenic mice," Journal of Experimental Medicine, vol. 194, no. 9, pp. 11951205, 2001.

[23] E. Quaglino, C. Mastini, A. Amici et al., "A better immune reaction to Erbb-2 tumors is elicited in mice by DNA vaccines encoding rat/human chimeric proteins," Cancer Research, vol. 70, no. 7, pp. 2604-2612, 2010.

[24] D. S. Ritchie, I. F. Hermans, J. M. Lumsden et al., "Dendritic cell elimination as an assay of cytotoxic T lymphocyte activity in vivo," Journal of Immunological Methods, vol. 246, no. 1-2, pp. 109-117, 2000.

[25] A. Smorlesi, F. Papalini, A. Amici et al., "Evaluation of different plasmid DNA delivery systems for immunization against HER2/neu in a transgenic murine model of mammary carcinoma," Vaccine, vol. 24, no. 11, pp. 1766-1775, 2006.

[26] G. J. Prud'homme, "DNA vaccination against tumors," Journal of Gene Medicine, vol. 7, no. 1, pp. 3-17, 2005.

[27] L. Timares, K. Mahmoud Safer, B. Qu, A. Takashima, and S. Albert Johnston, "Drug-inducible, dendritic cell-based genetic immunization," Journal of Immunology, vol. 170, no. 11, pp. 5483-5490, 2003.

[28] L. Timares, A. Takashima, and S. A. Johnston, "Quantitative analysis of the immunopotency of genetically transfected dendritic cells," Proceedings of the National Academy of Sciences of the United States of America, vol. 95, no. 22, pp. 13147-13152, 1998.

[29] M. B. M. Teunissen, M. Haniffa, and M. P. Collin, "Insight into the immunobiology of human skin and functional specialization of skin dendritic cell subsets to innovate intradermal vaccination design," Current Topics in Microbiology and Immunology, vol. 351, no. 1, pp. 25-76, 2012.

[30] B. Combadiere and C. Liard, "Transcutaneous and intradermal vaccination," Human Vaccines, vol. 7, no. 8, pp. 811-827, 2011.

[31] M. Kwissa, J. von Kampen, R. Zurbriggen, R. Glück, J. Reimann, and R. Schirmbeck, "Efficient vaccination by intradermal or intramuscular inoculation of plasmid DNA expressing hepatitis B surface antigen under desmin promoter/enhancer control," Vaccine, vol. 18, no. 22, pp. 2337-2344, 2000.

[32] M. J. McCluskie, C. L. B. Millan, R. A. Gramzinski et al., "Route and method of delivery of DNA vaccine influence immune responses in mice and non-human primates," Molecular Medicine, vol. 5, no. 5, pp. 287-300, 1999.

[33] W. Böhm, T. Mertens, R. Schirmbeck, and J. Reimann, "Routes of plasmid DNA vaccination that prime murine humoral and cellular immune responses," Vaccine, vol. 16, no. 9-10, pp. 949954, 1998. 
[34] F. Eriksson, T. Tötterman, A.-K. Maltais, P. Pisa, and J. Yachnin, "DNA vaccine coding for the rhesus prostate specific antigen delivered by intradermal electroporation in patients with relapsed prostate cancer," Vaccine, vol. 31, no. 37, pp. 3843-3848, 2013.

[35] U. R. Hengge, P. S. Walker, and J. C. Vogel, "Expression of naked DNA in human, pig, and mouse skin," The Journal of Clinical Investigation, vol. 97, no. 12, pp. 2911-2916, 1996.

[36] A. Gothelf and J. Gehl, "Gene electrotransfer to skin; review of existing literature and clinical perspectives," Current Gene Therapy, vol. 10, no. 4, pp. 287-299, 2010.

[37] L. Zhang, L. Li, G. A. Hofmann, and R. M. Hoffman, "Depthtargeted efficient gene delivery and expression in the skin by pulsed electric fields: an approach to gene therapy of skin aging and other diseases," Biochemical and Biophysical Research Communications, vol. 220, no. 3, pp. 633-636, 1996.

[38] L. Zhang, E. Nolan, S. Kreitschitz, and D. P. Rabussay, "Enhanced delivery of naked DNA to the skin by non-invasive in vivo electroporation," Biochimica et Biophysica Acta, vol. 1572, no. 1, pp. 1-9, 2002.

[39] B. M. Medi, S. Hoselton, R. B. Marepalli, and J. Singh, "Skin targeted DNA vaccine delivery using electroporation in rabbits I: efficacy," International Journal of Pharmaceutics, vol. 294, no. 1-2, pp. 53-63, 2005.

[40] G. Vandermeulen, K. Vanvarenberg, A. De Beuckelaer et al., "The site of administration influences both the type and the magnitude of the immune response induced by DNA vaccine electroporation," Vaccine, vol. 33, no. 28, pp. 3179-3185, 2015.

[41] K. Ito, K. Ito, N. Shinohara, and S. Kato, "DNA immunization via intramuscular and intradermal routes using a gene gun provides different magnitudes and durations on immune response," Molecular Immunology, vol. 39, no. 14, pp. 847-854, 2003.

[42] M. Spadaro, S. Lanzardo, C. Curcio, G. Forni, and F. Cavallo, "Immunological inhibition of carcinogenesis," Cancer Immunology, Immunotherapy, vol. 53, no. 3, pp. 204-216, 2004.

[43] E. Quaglino, S. Rolla, M. Iezzi et al., "Concordant morphologic and gene expression data show that a vaccine halts HER-2/neu preneoplastic lesions," Journal of Clinical Investigation, vol. 113, no. 5, pp. 709-717, 2004.

[44] P. Cappello, F. Triebel, M. Iezzi et al., "LAG-3 enables DNA vaccination to persistently prevent mammary carcinogenesis in HER-2/neu transgenic BALB/c mice," Cancer Research, vol. 63, no. 10, pp. 2518-2525, 2003.

[45] L. N. Klapper, N. Vaisman, E. Hurwitz, R. Pinkas-Kramarski, Y. Yarden, and M. Sela, "A subclass of tumor-inhibitory monoclonal antibodies to ErbB-2/HER2 blocks crosstalk with growth factor receptors," Oncogene, vol. 14, no. 17, pp. 2099-2109, 1997.

[46] M. Katsumata, T. Okudaira, A. Samanta et al., "Prevention of breast tumor development in vivo by downregulation of the Her2/neu receptor," Nature Medicine, vol. 1, Article ID 644648, pp. 644-648, 1995.

[47] Y. L. Yip and R. L. Ward, "Anti-ErbB-2 monoclonal antibodies and ErbB-2-directed vaccines," Cancer Immunology, Immunotherapy, vol. 50, no. 11, pp. 569-587, 2002.

[48] L. N. Klapper, H. Waterman, M. Sela, and Y. Yarden, “Tumorinhibitory antibodies to HER-2/ErbB2 may act by recruiting c$\mathrm{Cbl}$ and enhancing ubiquitination of HER-2," Cancer Research, vol. 60 , no. 13, pp. 3384-3388, 2000. 


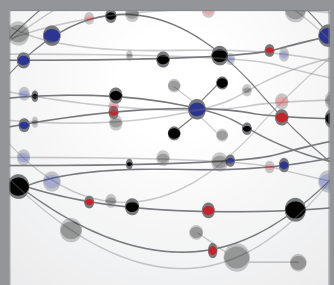

The Scientific World Journal
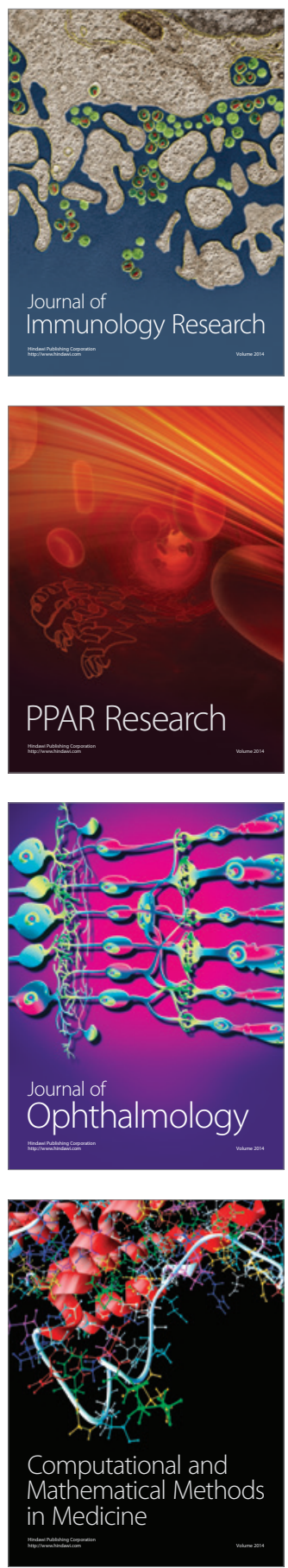

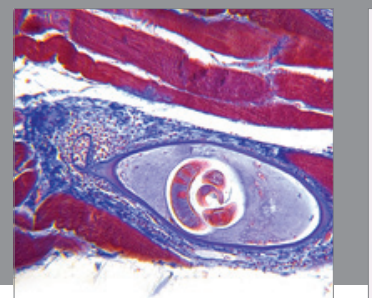

Gastroenterology

Research and Practice
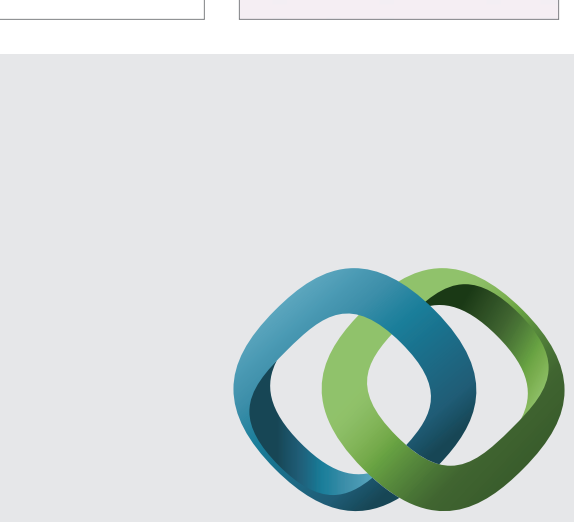

\section{Hindawi}

Submit your manuscripts at

http://www.hindawi.com
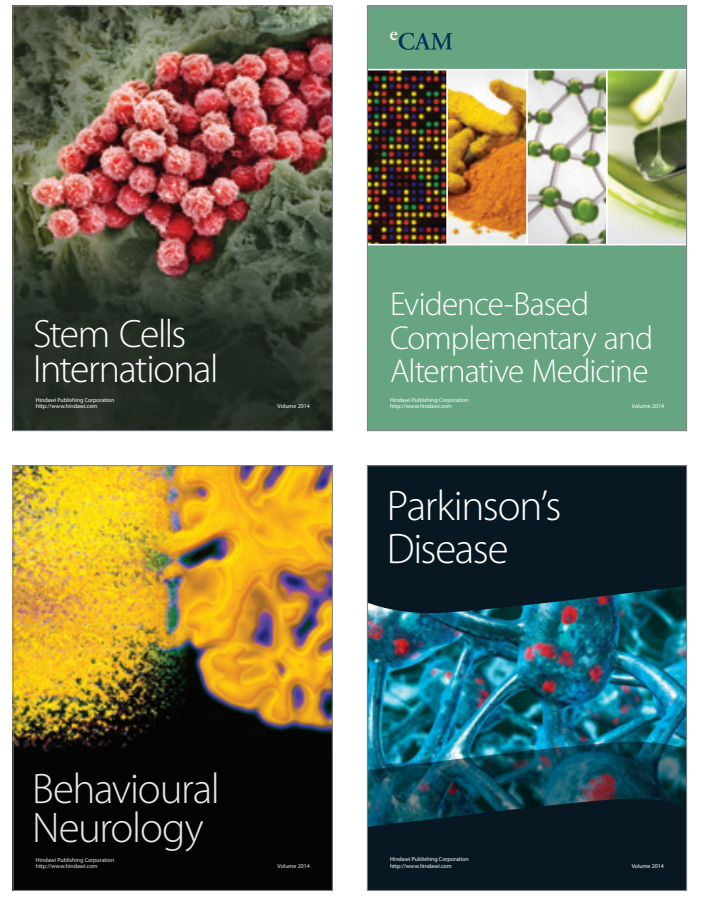
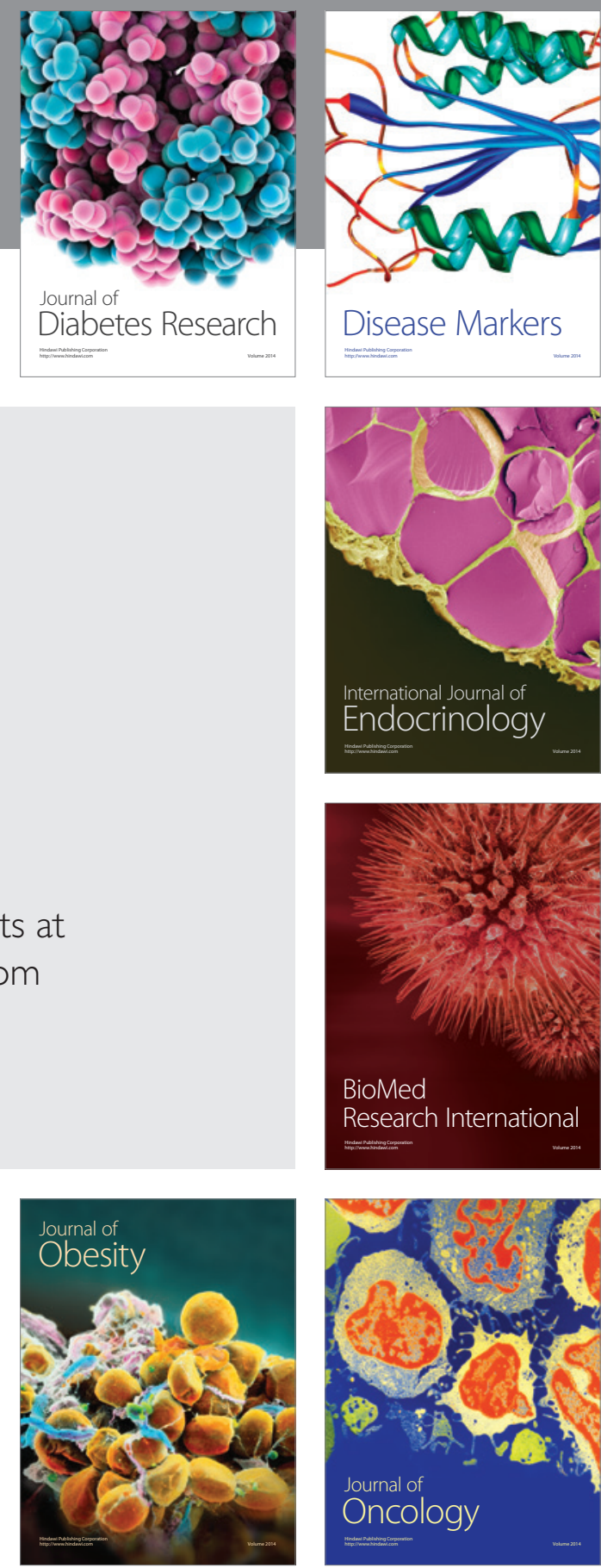

Disease Markers
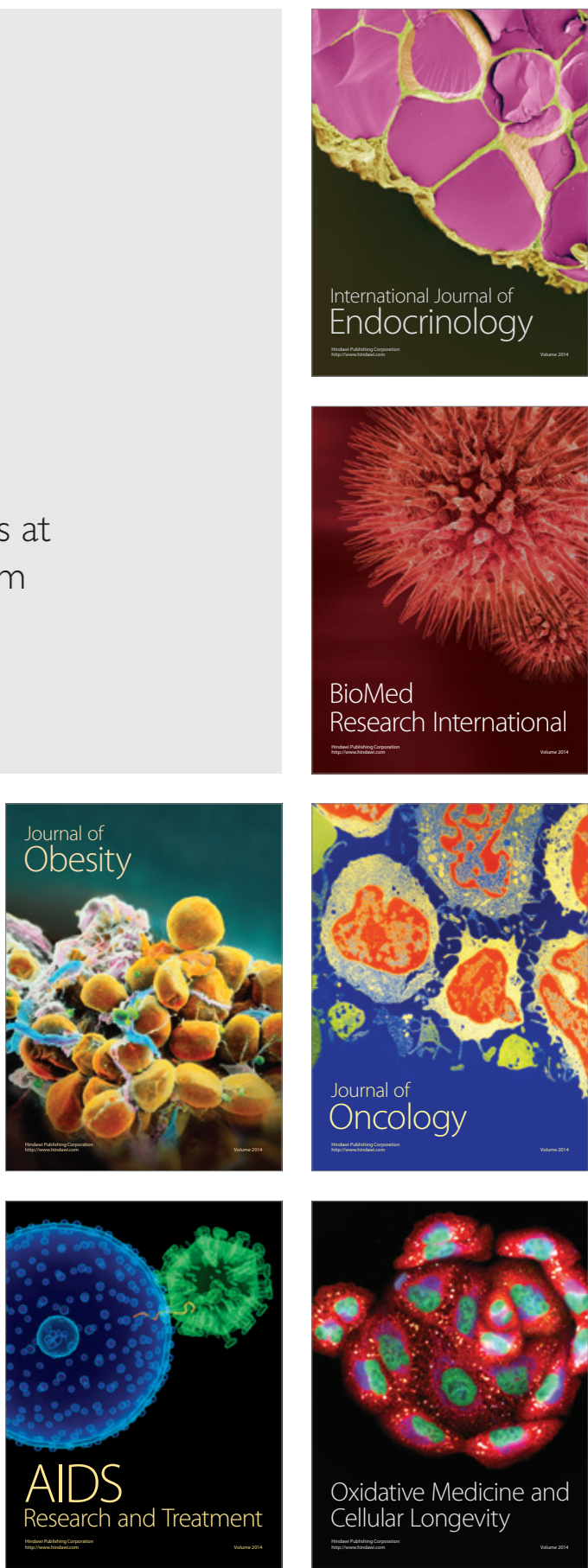